\title{
edoc
}

Institutional Repository of the University of Basel

University Library

Schoenbeinstrasse 18-20

CH-4056 Basel, Switzerland

http://edoc.unibas.ch/

Year: 2012

\section{Preconditioning of wavelet BEM by the incomplete Cholesky factorization}

Harbrecht, Helmut

Posted at edoc, University of Basel

Official URL: http://edoc.unibas.ch/dok/A6271911

Originally published as:

Harbrecht, Helmut. (2012) Preconditioning of wavelet BEM by the incomplete Cholesky factorization. Computing and visualization in science, Vol. 15, H. 6 , S. 319-329.

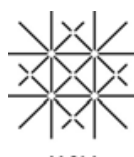




\title{
Preconditioning of Wavelet BEM by the Incomplete Cholesky Factorization
}

\author{
Helmut Harbrecht \\ Mathematisches Institut, Universität Basel \\ Rheinsprung 21, 4051 Basel, Switzerland \\ helmut.harbrecht@unibas.ch
}

\begin{abstract}
The present paper is dedicated to the preconditioning of boundary element matrices which are given in wavelet coordinates. We investigate the incomplete Cholesky factorization for a pattern which includes also the coefficients of all off-diagonal bands associated with the level-level-interactions. The pattern is chosen in such a way that the incomplete Cholesky factorization is computable in log-linear complexity. Numerical experiments are performed to quantify the effects of the proposed preconditioning.
\end{abstract}

\section{Introduction}

Various problems in science and engineering lead to boundary integral equations. In general such boundary integral equations are discretized by the boundary element method (BEM). For example, BEM is a favorable approach for the treatment of exterior boundary value problems, especially for problems in electrostatics and electromagnetics, or in case of the Helmholtz equation. Nevertheless, traditional discretizations will lead to linear systems with densely populated matrices. This feature makes the computation very costly in both respects, the computation time and computer memory requirements.

Over recent decades, several ideas for the efficient approximation of the discrete system have been developed. Most prominent examples of such methods are the fast multipole method [7], the panel clustering [9], the wavelet BEM [1, 4], and the 
hierarchical matrix approach [8]. These discretization methods end up with linear or almost linear complexity with respect to the number of boundary elements.

The present paper is concerned with wavelet BEM. A Galerkin discretization by wavelet bases yields quasi-sparse matrices, i.e., most matrix entries are negligible and can be treated as zero. Discarding the non-relevant matrix entries is called matrix compression. In [19] a fully discrete wavelet Galerkin method has been developed which produces approximate solutions within discretization accuracy in linear complexity.

If the boundary integral operator has an order different from zero, it acts on different length scales in a different way. This is well known to entail the linear systems to become more and more ill-conditioned when the level of resolution increases. Due to the explicit multilevel structure, properly scaled wavelet bases satisfy norm equivalences for a whole range of Sobolev spaces. This fact leads to a simple diagonal preconditioner. Since matrix-vector multiplications can be performed extremely fast due to the sparsity of the compressed system matrix, the linear system of equations can be rapidly solved.

However, despite of the preconditioning, the iterative solver often needs still a lot of iterations. There are many applications which require extremely good preconditioners. This is for example the case when the system has to be solved for several right hand sides as in shape optimization (e.g. [11]) or in inverse obstacle problems (e.g. [13]) to compute the local shape derivates. In the latter application, due to the so-called adjoint approach, the iterative solution has additionally to be very accurate to ensure the symmetry in the iteratively regularized Gauss-Newton method (IRGNM).

A further important application issues from the coupling of FEM and BEM. Here, the system matrix involve, besides the boundary element matrices, also finite element matrices. The whole system corresponds to a saddle point problem that involves operators of positive and negative order. Hence, preconditioning becomes an extremely important issue since a matrix-vector multiplication is quite expensive, see $[2,17,21]$. Other examples concerning continuum solvation models and uncertainty quantification are given in the numerical results in Section 6.

To improve the standard diagonal preconditioner we shall incorporate the block diagonals of the sub-matrices belonging to fixed level combinations of the ansatz and test wavelets. That way, also the interactions between different levels are considered. More generally, we develop an incomplete Cholesky factorization (ICF) which includes all block diagonal bands, where the width of the bands is controlled by a 
parameter. The larger the bandwidth parameter the more coefficients are included, which improves the preconditioning. We prove log-linear complexity of the ICF preconditioner and quantify it by numerical experiments. It turns out that the number of iterations decreases impressively.

The paper is organized as follows. Section 2 introduces the problem class under consideration. The wavelet bases and their properties are considered in Section 3. Section 4 briefly repeats the main features of the fully discrete wavelet Galerkin method from [19]. Then, in Section 5, the incomplete Cholesky factorization is developed. Section 6 is devoted to numerical experiments. Finally, Section 7 contains concluding remarks.

In the following, in order to avoid the repeated use of generic but unspecified constants, by $C \lesssim D$ we mean that $C$ can be bounded by a multiple of $D$, independently of parameters which $C$ and $D$ may depend on. Obviously, $C \gtrsim D$ is defined as $D \lesssim C$, and $C \sim D$ as $C \lesssim D$ and $C \gtrsim D$.

\section{Problem Formulation and Preliminaries}

We consider a boundary integral equation on the closed boundary surface $\Gamma:=\partial \Omega$ of a three-dimensional domain $\Omega \subset \mathbb{R}^{3}$ :

$$
(\mathcal{A} u)(\mathbf{x})=\int_{\Gamma} k(\mathbf{x}, \mathbf{y}) u(\mathbf{y}) \mathrm{d} \sigma_{\mathbf{y}}=f(\mathbf{x}), \quad \mathbf{x} \in \Gamma .
$$

Herein, the boundary integral operator $\mathcal{A}: H^{q}(\Gamma) \rightarrow H^{-q}(\Gamma)$ is assumed to be a symmetric and bijective operator of order $2 q \neq 0$. The kernel functions under consideration are supposed to be smooth as functions in the variables $\mathbf{x}$ and $\mathbf{y}$, apart from the diagonal $\{(\mathbf{x}, \mathbf{y}) \in \Gamma \times \Gamma: \mathbf{x}=\mathbf{y}\}$ and may have a singularity on the diagonal. Such kernel functions arise, for instance, by applying a boundary integral formulation to a second order elliptic problem [29, 33]. In general, they decay like a negative power of the distance of the arguments which depends on the order $2 q$ of the operator. More precisely, there holds

$$
\left|\partial_{\mathbf{x}}^{\boldsymbol{\alpha}} \partial_{\mathbf{y}}^{\boldsymbol{\beta}} k(\mathbf{x}, \mathbf{y})\right| \leq c_{\boldsymbol{\alpha}, \boldsymbol{\beta}}\|\mathbf{x}-\mathbf{y}\|^{-2-2 q-|\boldsymbol{\alpha}|-|\boldsymbol{\beta}|} .
$$

We will assume that the boundary $\Gamma$ is represented by piecewise parametric mappings. Let $\square:=[0,1]^{2}$ denote the unit square. We subdivide the given manifold into several patches

$$
\Gamma=\bigcup_{i=1}^{M} \Gamma_{i}, \quad \Gamma_{i}=\gamma_{i}(\square), \quad i=1,2, \ldots, M
$$



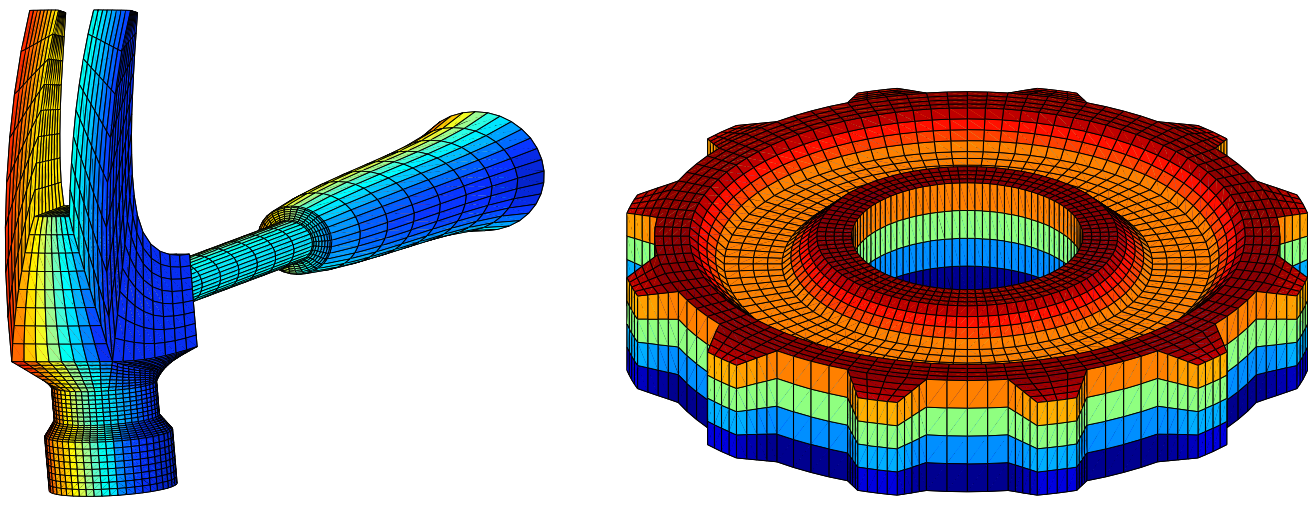

Figure 2.1: Parameterizations of a hammer and a gearwheel.

such that each $\gamma_{i}: \square \rightarrow \Gamma_{i}$ defines a diffeomorphism of $\square$ onto $\Gamma_{i}$. The intersection $\Gamma_{i} \cap \Gamma_{i^{\prime}}, i \neq i^{\prime}$, of the patches $\Gamma_{i}$ and $\Gamma_{i^{\prime}}$ is supposed to be either $\emptyset$, a common edge, or a common vertex.

A mesh of level $j$ on $\Gamma$ is induced by dyadic subdivisions of depth $j$ of the unit square into $4^{j}$ squares. This generates $4^{j} M$ elements (or elementary domains). In order to get a regular mesh of $\Gamma$, the parametric representation is supposed to be globally continuous.

The surface representation is in contrast to the common approximation of surfaces by panels. It has the advantage that the rate of convergence is not limited by approximation. Technical surfaces generated by tools from Computer Aided Design (CAD) are often represented in the present form.

The most common geometry representation in CAD is defined by the IGES (Initial Graphics Exchange Specification) standard. Here, the initial CAD object is a solid, bounded by a closed surface that is given as a collection of parametric surfaces which can be trimmed or untrimmed. An untrimmed surface is already a four-sided patch, parameterized over a rectangle. Whereas, a trimmed surface is just a piece of a supporting untrimmed surface, described by boundary curves. There are several representations of the parameterizations including B-splines, NURBS (nonuniform rational B-Splines), surfaces of revolution, and tabulated cylinders [22].

In [15], an algorithm has been developed to decompose a technical surface, described in the IGES format, into a collection of parameterized four-sided patches, fulfilling all the above requirements. In $[14,16]$, the algorithm has been extended to molecular surfaces. Figure 2.1 visualizes two parameterizations which satisfy the present requirements. 


\section{Wavelets and Multiresolution Analysis}

In general, a multiresolution analysis consists of a nested family of finite dimensional subspaces

$$
V_{0} \subset V_{1} \subset \cdots \subset V_{j} \subset V_{j+1} \cdots \subset \cdots \subset L^{2}(\Gamma),
$$

such that $\operatorname{dim} V_{j} \sim 4^{j}$ and $\overline{\bigcup_{j \geq 0} V_{j}}=L^{2}(\Gamma)$. Each space $V_{j}$ is defined by a singlescale basis $\Phi_{j}=\left\{\phi_{j, \mathbf{k}}: \mathbf{k} \in \Delta_{j}\right\}$, i.e., $V_{j}=\operatorname{span} \Phi_{j}$, where $\Delta_{j}$ denotes a suitable index set with cardinality $\left|\Delta_{j}\right| \sim 4^{j}$. It is convenient to identify bases with row vectors, such that, for $\mathbf{v}=\left[v_{k}\right]_{k \in \Delta_{j}} \in \ell^{2}\left(\Delta_{j}\right)$, the function $v_{j}=\Phi_{j} \mathbf{v}$ is defined as $v_{j}=\sum_{k \in \Delta_{j}} v_{k} \varphi_{j, k}$. A final requirement is that the bases $\Phi_{j}$ are uniformly stable, i.e., $\|\mathbf{v}\|_{\ell^{2}\left(\Delta_{j}\right)} \sim\left\|\Phi_{j} \mathbf{v}\right\|_{L^{2}(\Gamma)}$ for all $\mathbf{v} \in \ell^{2}\left(\Delta_{j}\right)$ uniformly in $j$. Furthermore, the single-scale bases satisfy the locality condition diam $\operatorname{supp} \phi_{j, \mathbf{k}} \sim 2^{-j}$.

Additional properties of the spaces $V_{j}$ are required for using them as trial spaces in a Galerkin scheme. The trial spaces shall have approximation order $d \in \mathbb{N}$ and regularity $\gamma>0$, that is

$$
\begin{gathered}
\gamma=\sup \left\{s \in \mathbb{R}: V_{j} \subset H^{s}(\Gamma)\right\}, \\
d=\sup \left\{s \in \mathbb{R}: \inf _{v_{j} \in V_{j}}\left\|v-v_{j}\right\|_{L^{2}(\Gamma)} \lesssim 2^{-j s}\|v\|_{H^{s}(\Gamma)}\right\} .
\end{gathered}
$$

Note that conformity of the Galerkin scheme induces $\gamma>q$.

Instead of using only a single-scale $j$, the idea of wavelet concepts is to keep track to the increment of information between two adjacent scales $j-1$ and $j$. Since $V_{j-1} \subset V_{j}$, one decomposes $V_{j}=V_{j-1} \oplus W_{j}$ with some complementary space $W_{j}$, $W_{j} \cap V_{j-1}=\{0\}$, not necessarily orthogonal to $V_{j-1}$. Of practical interest are the bases of the complementary spaces $W_{j}$ in $V_{j}$

$$
\Psi_{j}=\left\{\psi_{j, \mathbf{k}}: \mathbf{k} \in \nabla_{j}:=\Delta_{j} \backslash \Delta_{j-1}\right\}
$$

It is supposed that the collections $\Phi_{j-1} \cup \Psi_{j}$ are also uniformly stable bases of $V_{j}$. If $\Psi=\bigcup_{j \geq 0} \Psi_{j}$, where $\Psi_{0}:=\Phi_{0}$, is a Riesz-basis of $L_{2}(\Gamma)$, it is called a wavelet basis. We assume the functions $\psi_{j, \mathbf{k}}$ to be local with respect to the corresponding scale $j$, i.e., diam $\operatorname{supp} \psi_{j, \mathbf{k}} \sim 2^{-j}$, and we normalize them such that $\left\|\psi_{j, \mathbf{k}}\right\|_{L_{2}(\Gamma)} \sim 1$.

At first glance it would be very convenient to deal with a single orthonormal system of wavelets. But it was shown in $[4,6,32]$ that orthogonal wavelets are not completely appropriate for the efficient solution of boundary integral equations. For that reason we use biorthogonal wavelet bases. Then, we have also a biorthogonal, or dual, multiresolution analysis, i.e., dual single-scale bases $\widetilde{\Phi}_{j}=\left\{\widetilde{\phi}_{j, \mathbf{k}}: \mathbf{k} \in \Delta_{j}\right\}$ and wavelets 
$\widetilde{\Psi}_{j}=\left\{\widetilde{\psi}_{j, \mathbf{k}}: \mathbf{k} \in \nabla_{j}\right\}$ which are coupled to the primal ones via $\left(\Phi_{j}, \widetilde{\Phi}_{j}\right)_{L^{2}(\Gamma)}=\mathbf{I}$ and $\left(\Psi_{j}, \widetilde{\Psi}_{j}\right)_{L^{2}(\Gamma)}=\mathbf{I}$. The associated spaces $\widetilde{V}_{j}:=\operatorname{span} \widetilde{\Phi}_{j}$ and $\widetilde{W}_{j}:=\operatorname{span} \widetilde{\Psi}_{j}$ satisfy

$$
V_{j-1} \perp \widetilde{W}_{j}, \quad \widetilde{V}_{j-1} \perp W_{j} .
$$

Also the dual spaces shall have some approximation order $\widetilde{d} \in \mathbb{N}$ and regularity $\widetilde{\gamma}>0$.

Denoting likewise to the primal side $\widetilde{\Psi}=\bigcup_{j \geq 0} \widetilde{\Psi}_{j}$, where $\widetilde{\Psi}_{0}:=\widetilde{\Phi}_{0}$, then every $v \in L^{2}(\Gamma)$ has a unique representation $v=\widetilde{\Psi}(v, \Psi)_{L^{2}(\Gamma)}=\Psi(v, \widetilde{\Psi})_{L^{2}(\Gamma)}$. Moreover, there hold the well known norm equivalences [3, 23]

$$
\begin{array}{ll}
\|v\|_{H^{t}(\Gamma)}^{2} \sim \sum_{j \geq 0} 2^{2 j t} \sum_{k \in \nabla_{j}}\left\|\left(v, \widetilde{\psi}_{j, \mathbf{k}}\right)_{L^{2}(\Gamma)}\right\|_{\ell^{2}\left(\nabla_{j}\right)}^{2}, \quad t \in(-\widetilde{\gamma}, \gamma), \\
\|v\|_{H^{t}(\Gamma)}^{2} \sim \sum_{j \geq 0} 2^{2 j t} \sum_{k \in \nabla_{j}}\left\|\left(v, \psi_{j, \mathbf{k}}\right)_{L^{2}(\Gamma)}\right\|_{\ell^{2}\left(\nabla_{j}\right)}^{2}, \quad t \in(-\gamma, \widetilde{\gamma}) .
\end{array}
$$

The relation (3.4) implies that the wavelets provide vanishing moments of order $\widetilde{d}$

$$
\left|\left(v, \psi_{j, \mathbf{k}}\right)_{L^{2}(\Gamma)}\right| \lesssim 2^{-j(1+\widetilde{d})}|v|_{W^{\tilde{d}, \infty}\left(\operatorname{supp} \psi_{j, \mathbf{k}}\right)} .
$$

Here $|v|_{W^{\widetilde{d}, \infty}(\Omega)}:=\sup _{|\boldsymbol{\alpha}|=\widetilde{d}}\left\|\partial^{\alpha} v\right\|_{L^{\infty}(\Omega)}$ denotes the semi-norm in $W^{\widetilde{d}, \infty}(\Omega)$. We refer to [3] for further details.

Piecewise constant and bilinear wavelets which provide the above properties have been constructed in $[18,20]$. In the rest of the paper we will denote the wavelet basis of $V_{J}$ by $\Psi_{J}=\left\{\psi_{\lambda}: \lambda \in \nabla_{J}\right\}$, where the multi-index $\lambda=(j, \mathbf{k})$ incorporates the scale $j=|\lambda|$ and the spatial location $\mathbf{k}=\mathbf{k}(\lambda)$.

\section{Wavelet Galerkin BEM}

We shall be concerned with the Galerkin method for the solution of the given boundary integral equation (2.1): find $u_{J} \in V_{J}$ which solves the variational problem

$$
\left(\mathcal{A} u_{J}, v_{J}\right)_{L^{2}(\Gamma)}=\left(f, v_{J}\right)_{L^{2}(\Gamma)} \quad \text { for all } \quad v_{J} \in V_{J} .
$$

Traditionally this equation is discretized by the single-scale basis of $V_{J}$ which yields a densely populated system matrix. This means that, if $N_{J} \sim 4^{J}$ denotes the number of basis functions in the space $V_{J}$, the system matrix contains $\mathcal{O}\left(N_{J}^{2}\right)$ nonzero matrix coefficients. Contrastingly, if we use a Galerkin discretization in wavelet coordinates, 
the matrix becomes quasi-sparse. In fact, by combining (2.2) and (3.6), we arrive at the decay estimate

$$
\left(\mathcal{A} \psi_{\lambda^{\prime}}, \psi_{\lambda}\right)_{L^{2}(\Gamma)} \lesssim \frac{2^{-\left(|\lambda|+\left|\lambda^{\prime}\right|\right)(1+\widetilde{d})}}{\operatorname{dist}\left(\Omega_{\lambda}, \Omega_{\lambda^{\prime}}\right)^{2(1+q+\widetilde{d})}}
$$

which is the main foundation of compression estimates [4]. Herein, $\Omega_{\lambda}:=\operatorname{conv}_{\Gamma} \operatorname{supp} \psi_{\lambda}$ and $\Omega_{\lambda^{\prime}}:=\operatorname{conv}_{\Gamma} \operatorname{supp} \psi_{\lambda}$ denote the convex hulls of the supports of the wavelets $\psi_{\lambda}$ and $\psi_{\lambda^{\prime}}$ relative to the surface $\Gamma$, respectively.

Based on (4.7), we can set all matrix entries to zero, for which the distance of the supports between the associated trial and test functions is larger than a level dependent cut-off parameter $\mathcal{B}_{j, j^{\prime}}$. Further compression, reflected by a cut-off parameter $\mathcal{B}_{j, j^{\prime}}^{s}$, is achieved by neglecting some of those matrix entries, for which the corresponding trial and test functions have overlapping supports.

To formulate this result, we introduce the abbreviation $\Omega_{\lambda}^{s}:=\operatorname{sing} \operatorname{supp} \psi_{\lambda}$ which denotes the singular support of the wavelet $\psi_{\lambda}$, i.e., that subset of $\Gamma$ where the wavelet is not smooth.

Theorem 4.1 (A-priori compression [4]). Let $\Omega_{\lambda}$ and $\Omega_{\lambda}^{s}$ be given as above and define the compressed system matrix $\mathbf{A}_{J}$, corresponding to the boundary integral operator $\mathcal{A}$, by

$$
\left[\mathbf{A}_{J}\right]_{\lambda, \lambda^{\prime}}:=\left\{\begin{aligned}
0, & \operatorname{dist}\left(\Omega_{\lambda}, \Omega_{\lambda^{\prime}}\right)>\mathcal{B}_{|\lambda|,\left|\lambda^{\prime}\right|} \text { and }|\lambda|,\left|\lambda^{\prime}\right|>0 \\
0, & \operatorname{dist}\left(\Omega_{\lambda}, \Omega_{\lambda^{\prime}}\right) \leq 2^{-\min \left\{|\lambda|,\left|\lambda^{\prime}\right|\right\}} \text { and } \\
& \operatorname{dist}\left(\Omega_{\lambda}^{s}, \Omega_{\lambda^{\prime}}\right)>\mathcal{B}_{|\lambda|,\left|\lambda^{\prime}\right|}^{s} \text { if }\left|\lambda^{\prime}\right|>|\lambda| \geq 0 \\
& \operatorname{dist}\left(\Omega_{\lambda}, \Omega_{\lambda^{\prime}}^{s}\right)>\mathcal{B}_{|\lambda|,\left|\lambda^{\prime}\right|}^{s} \text { if }|\lambda|>\left|\lambda^{\prime}\right| \geq 0 \\
\left(\mathcal{A} \psi_{\lambda^{\prime}}, \psi_{\lambda}\right)_{L^{2}(\Gamma)}, & \text { otherwise. }
\end{aligned}\right.
$$

Fixing

$$
a>1, \quad d<\delta<\widetilde{d}+2 q,
$$

the cut-off parameters $\mathcal{B}_{j, j^{\prime}}$ and $\mathcal{B}_{j, j^{\prime}}^{s}$ are set as follows

$$
\begin{array}{r}
\mathcal{B}_{j, j^{\prime}}=a \max \left\{2^{-\min \left\{j, j^{\prime}\right\}}, 2^{\frac{2 J(\delta-q)-\left(j+j^{\prime}\right)(\delta+\widetilde{d})}{2(\tilde{d}+q)}}\right\}, \\
\mathcal{B}_{j, j^{\prime}}^{s}=a \max \left\{2^{-\max \left\{j, j^{\prime}\right\}}, 2^{\frac{2 J(\delta-q)-\left(j+j^{\prime}\right) \delta-\max \left\{j, j^{\prime}\right\} \widetilde{d}}{\tilde{d}+2 q}}\right\} .
\end{array}
$$

Then, the system matrix $\mathbf{A}_{J}$ has only $\mathcal{O}\left(N_{J}\right)$ nonzero coefficients. Moreover, the error estimate

$$
\left\|u-u_{J}\right\|_{H^{2 q-d}(\Gamma)} \lesssim 2^{-2 J(d-q)}\|u\|_{H^{d}(\Gamma)}
$$



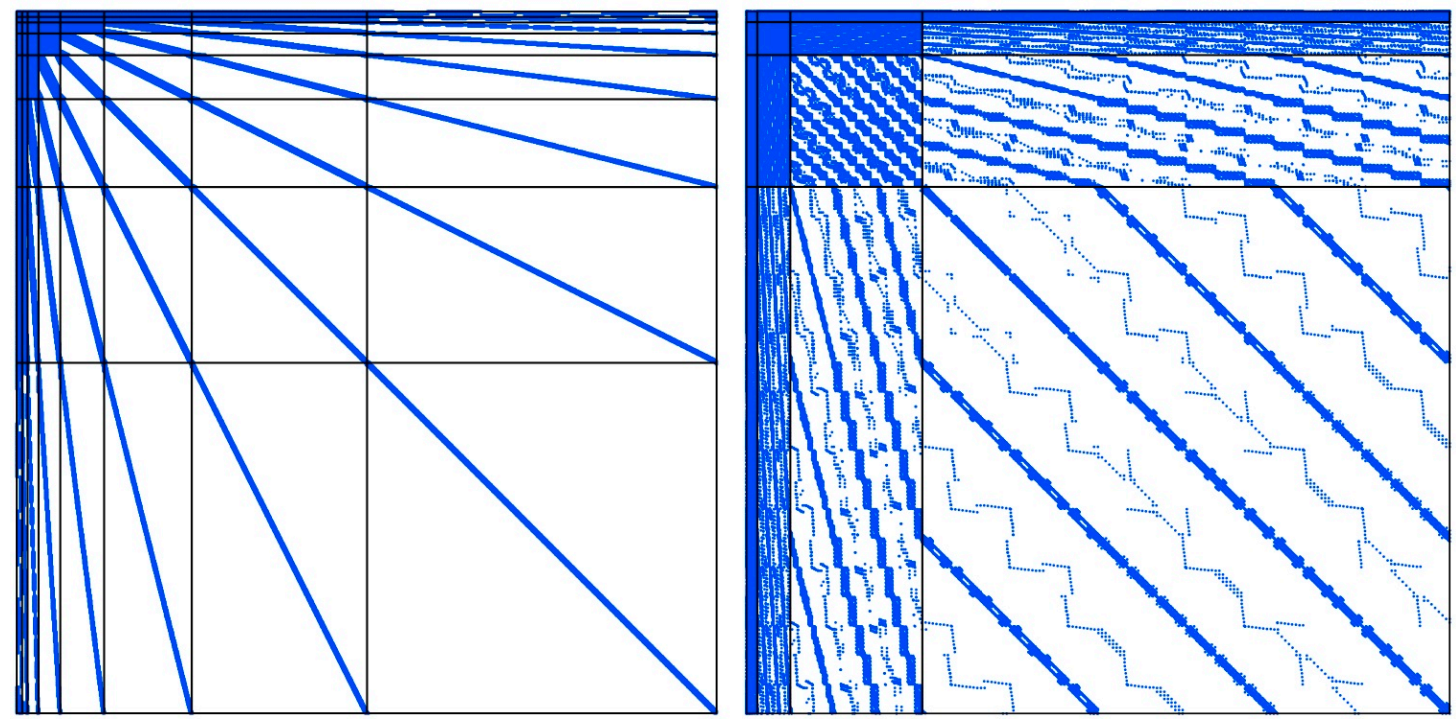

Figure 4.2: Compression pattern in case of a circle (left) and a sphere (right).

holds for the solution $u_{J}$ of the compressed Galerkin system provided that $u$ and $\Gamma$ are sufficiently regular.

The compressed system matrix can be assembled in linear complexity if one employs the exponentially convergent $h p$-quadrature method proposed in [19]. Moreover, for performing faster matrix-vector multiplications, an additional a-posteriori compression might be applied which reduces again the number of nonzero coefficients by a factor 2-5 [4]. The pattern of the compressed system matrix exhibit the typical finger structure, see (4.2).

If the boundary integral operator $\mathcal{A}$ has an order $q$ different from 0 , the compressed system matrix $\mathbf{A}_{J}$ becomes more and more ill-conditioned when the level $J$ increases. More precisely, the condition number of the system matrix will asymptotically grow like $2^{2 J|q|}$ as the level $J$ increases. However, as an immediate consequence of the norm equivalences (3.5) of wavelet bases, normalizing the wavelets relative to the energy norm leads to uniformly bounded condition numbers.

Theorem 4.2 (Preconditioning $[5,32]$ ). Let the diagonal matrix $\mathbf{D}_{J}^{r}$ defined by

$$
\left[\mathbf{D}_{J}^{r}\right]_{\lambda, \lambda^{\prime}}=2^{r|\lambda|} \delta_{\lambda, \lambda^{\prime}}, \quad \lambda, \lambda^{\prime} \in \nabla_{J}
$$

Then, if the regularity $\widetilde{\gamma}$ of the dual wavelets satisfies $\widetilde{\gamma}>-q$, the diagonal matrix $\mathbf{D}_{J}^{2 q}$ defines an asymptotically optimal preconditioner to $\mathbf{A}_{J}$, i.e.,

$$
\operatorname{cond}_{\ell^{2}}\left(\mathbf{D}_{J}^{-q} \mathbf{A}_{J} \mathbf{D}_{J}^{-q}\right) \sim 1
$$


Remark 4.3. The entries on the main diagonal of $\mathbf{A}_{J}$ satisfy

$$
\left(\mathcal{A} \psi_{\lambda}, \psi_{\lambda}\right)_{L^{2}(\Gamma)} \sim 2^{2|q||\lambda|} .
$$

Therefore, the above preconditioning can be replaced by a diagonal scaling. In fact, the diagonal scaling improves and even simplifies the standard wavelet preconditioning.

\section{Incomplete Cholesky Factorization}

Often the above diagonal preconditioner does not lead to satisfactory results. The idea to improve the wavelet preconditioning is to use not only the main diagonal of the system matrix as a preconditioner, but also all block diagonals of the submatrices $\mathbf{A}_{j, j^{\prime}}:=\left[\left(\mathcal{A} \psi_{\lambda^{\prime}}, \psi_{\lambda}\right)_{L^{2}(\Gamma)}\right]_{|\lambda|=j\left|\lambda^{\prime}\right|=j^{\prime}}$. More generally, we shall compute the incomplete Cholesky factorization $\mathbf{A}_{J} \approx \mathbf{L}_{J} \mathbf{L}_{J}^{T}$ with respect to a matrix pattern which is finger structured like the compressed system matrix. For a suitable matrix pattern $\mathcal{I} \subset \nabla_{J} \times \nabla_{J}$, the incomplete Cholesky factorization is given by the following algorithm:

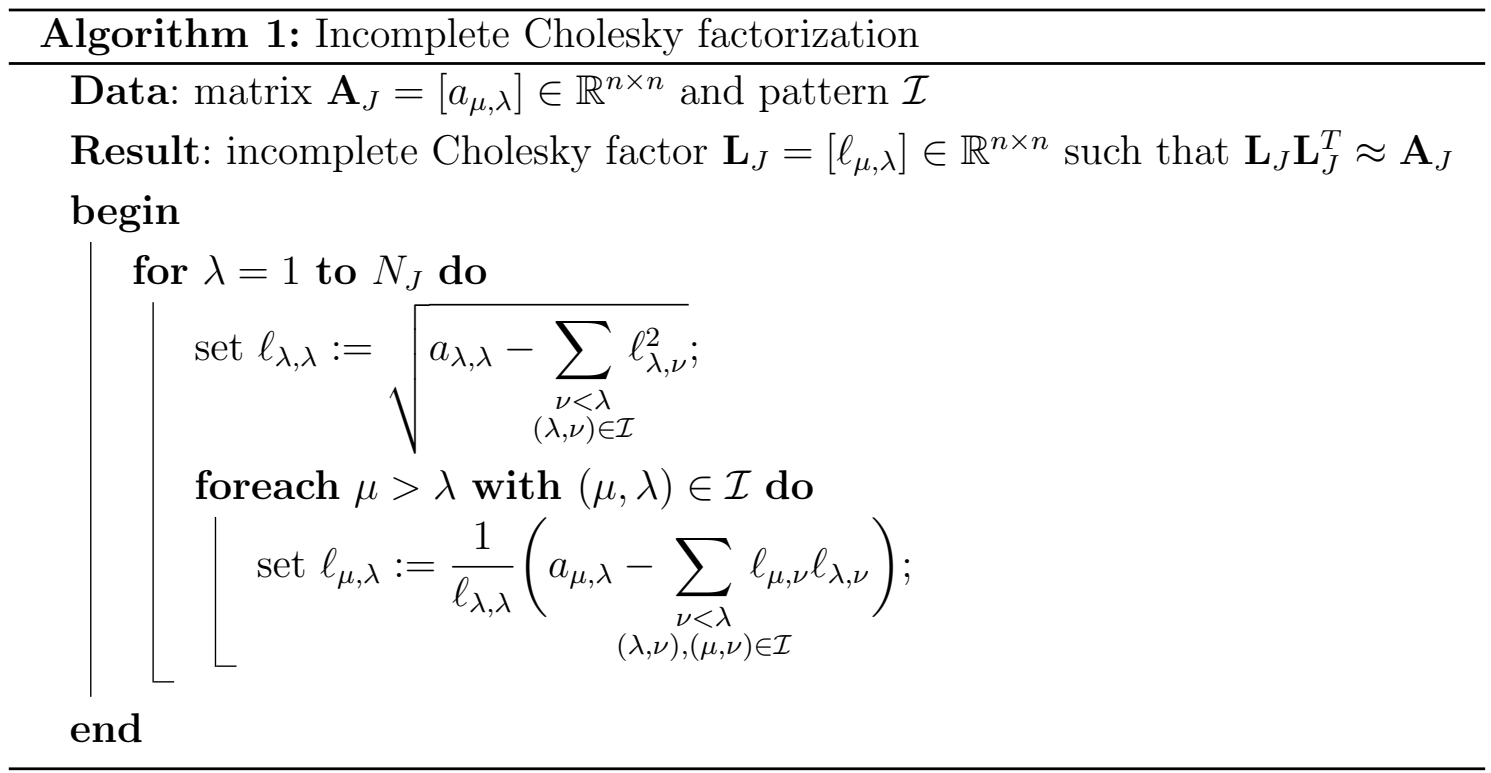

We shall demonstrate at first that we cannot simply compute the incomplete Cholesky factorization with respect to the pattern of the compressed system matrix $\mathbf{A}_{J}$ since it is too expensive. To this end, let for sake of simplicity the pattern $\mathcal{I}$ be just the main diagonal band of the compressed system matrix $\mathbf{A}_{J}$. In the main diagonal blocks of $\mathbf{A}_{J}$, only the first compression is active, where the associated cut-off parameter (cf. (4.9) and (4.10)) satisfies

$$
\mathcal{B}_{j, j}=a 2^{-J} 2^{(J-j) M} \quad \text { where } \quad 1<M:=\frac{\delta+\widetilde{d}}{\widetilde{d}+q}<2 .
$$



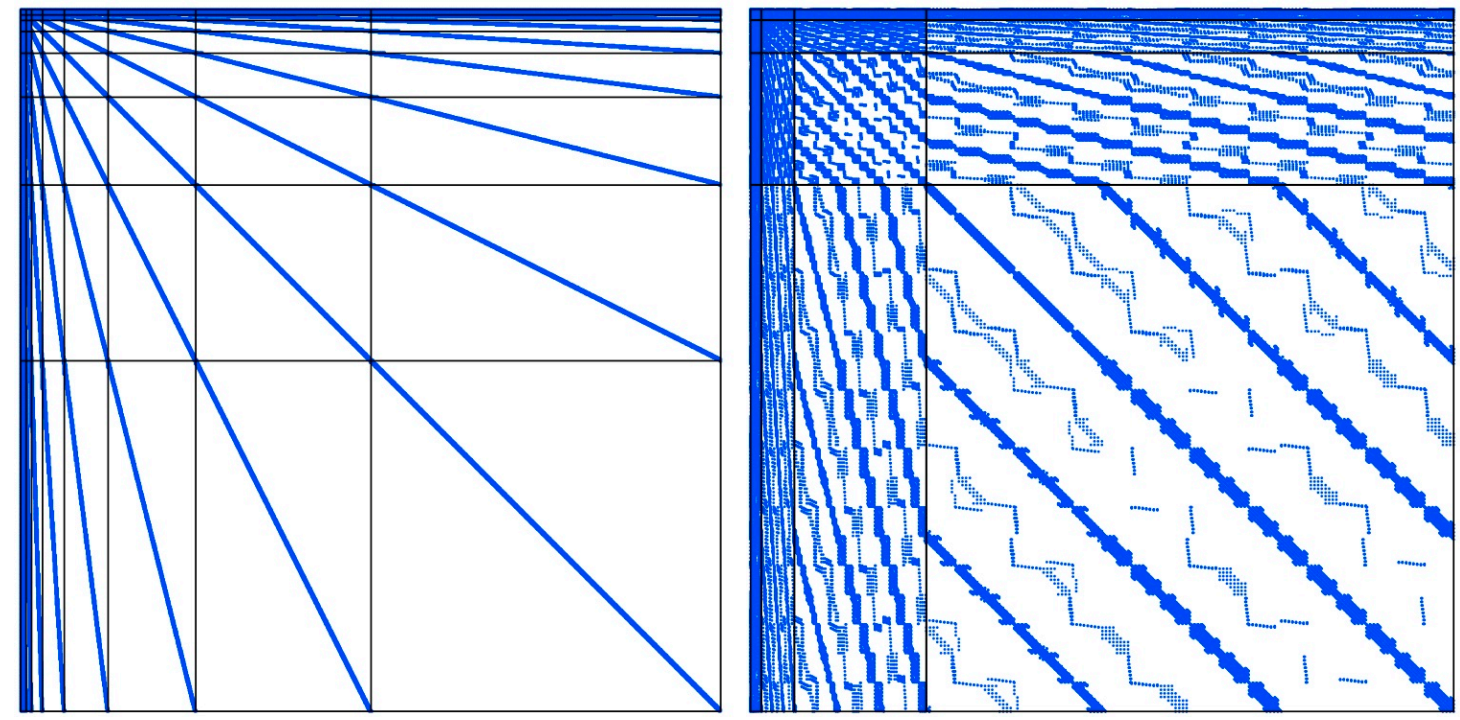

Figure 5.3: The pattern of the incomplete Cholesky factorization in case of a circle (left) and a sphere (right).

Thus, the main diagonal band of the compressed system matrix owns $\mathcal{O}\left(\left[2^{j} \mathcal{B}_{j, j}\right]^{2}\right)=$ $\mathcal{O}\left(4^{(J-j)(M-1)}\right)$ coefficients per row on the level $j$. Consequently, since there are $\mathcal{O}\left(4^{j}\right)$ wavelets on level $j$, the computational effort of the associated incomplete Cholesky factorization would be

$$
\sum_{j=0}^{J} 4^{j}\left(\left[2^{j} \mathcal{B}_{j, j}\right]^{2}\right)^{2} \sim \sum_{j=0}^{J} 4^{j} 4^{(J-j)(2 M-2)} \sim 4^{J} \sum_{j=0}^{J} 4^{(J-j)(2 M-3)} .
$$

Since it holds $2 M>3$ for realistic choices of $(d, \widetilde{d})$, we arrive at the complexity $\mathcal{O}\left(4^{J(2 M-2)}\right)$ which is always less than a quadratic complexity, but significantly higher than a linear complexity.

This reasoning shows that we need another strategy to define the pattern of incomplete Cholesky factorization. In fact, the problem is that the bandwidth of the fingers increases when the level decreases (cf. Figure 4.2). We shall thus choose a fixed bandwidth of the fingers. The resulting pattern is shown in Figure 5.3.

Theorem 5.1. Define the pattern of the incomplete Cholesky factorization as that subset $\mathcal{I}$ of $\nabla_{J} \times \nabla_{J}$ which satisfies

$$
\operatorname{dist}\left(\Omega_{\lambda}, \Omega_{\lambda^{\prime}}\right) \leq \mathcal{C}_{|\lambda|,\left|\lambda^{\prime}\right|}:=2^{-\min \left\{|\lambda|,\left|\lambda^{\prime}\right|\right\}} b, \quad b \geq 0
$$

Then, the cost of computing of the incomplete Cholesky factorization is $\mathcal{O}\left(J^{2} N_{J}\right)$. 
Proof. We shall first estimate the work to compute the $\lambda$-th column of the incomplete Cholesky factorization. According to Algorithm 1, for a single coefficient $\ell_{\mu, \lambda}$, the work is bounded by the sum of the numbers of all nonzero coefficients $\ell_{\mu, \nu}$ of the $\mu$-th and $\ell_{\lambda, \nu}$ of the $\lambda$-th row vector with $\nu \leq \lambda$. This number has then to be multiplied with the number of nonzero coefficients found in the $\lambda$-th column.

Let $\mathbf{L}_{j, j^{\prime}}$ denote that matrix block incomplete Cholesky factorization which consists of the coefficients $\ell_{\lambda, \lambda^{\prime}}$ with $|\lambda|=j$ and $\left|\lambda^{\prime}\right|=j^{\prime}$. The block is empty if $j<j^{\prime}$ since $\mathbf{L}_{J}$ is lower triangular. If $j \geq j^{\prime}$, then the block contains only $\mathcal{O}\left(\left[2^{j-j^{\prime}}\right]^{2}\right)$ coefficients per column with $\operatorname{dist}\left(\Omega_{\lambda}, \Omega_{\lambda^{\prime}}\right)=0$ and $\mathcal{O}\left(\left[2^{j} \mathcal{C}_{j, j^{\prime}}\right]^{2}\right)$ coefficients per column with $0 \neq \operatorname{dist}\left(\Omega_{\lambda}, \Omega_{\lambda^{\prime}}\right) \leq \mathcal{C}_{j, j^{\prime}}$. Thus, the number of nonzero coefficients of the $\lambda$-th column vector of $\mathbf{L}_{J}$ is

$$
\operatorname{nnz}\left(\ell_{:, \lambda}\right) \lesssim \sum_{j=|\lambda|}^{J}\left(4^{j-|\lambda|}+4^{j} \mathcal{C}_{j,|\lambda|}^{2}\right) \sim \sum_{j=|\lambda|}^{J} 4^{j-|\lambda|} \sim 4^{J-|\lambda|}
$$

Next, we count the number of nonzero coefficients which enter the computation of $\ell_{\mu, \lambda}$. In each block $\mathbf{L}_{j, j^{\prime}}$ with $j \geq j^{\prime} \geq|\lambda|$, we find only $\mathcal{O}(1)$ coefficients per row with $\operatorname{dist}\left(\Omega_{\lambda}, \Omega_{\lambda^{\prime}}\right)=0$ and $\mathcal{O}\left(\left[2^{j^{\prime}} \mathcal{C}_{j, j^{\prime}}\right]^{2}\right)$ coefficients per row with $0 \neq \operatorname{dist}\left(\Omega_{\lambda}, \Omega_{\lambda^{\prime}}\right) \leq \mathcal{C}_{j, j^{\prime}}$. This leads to

$$
\begin{aligned}
\operatorname{nnz}\left(\ell_{\mu, 1: \lambda}\right) & \lesssim \sum_{j^{\prime}=0}^{|\lambda|}\left(1+4^{j^{\prime}} \mathcal{C}_{|\mu|, j^{\prime}}^{2}\right) \sim \sum_{j^{\prime}=0}^{|\lambda|} 1 \sim|\lambda| \\
\operatorname{nnz}\left(\ell_{\lambda, 1: \lambda}\right) & \lesssim \sum_{j^{\prime}=0}^{|\lambda|}\left(1+4^{j^{\prime}} \mathcal{C}_{|\lambda|, j^{\prime}}^{2}\right) \sim \sum_{j^{\prime}=0}^{|\lambda|} 1 \sim|\lambda| .
\end{aligned}
$$

Hence, the work to compute the complete $\lambda$-th column vector of the incomplete Cholesky factorization is bounded by

$$
\operatorname{nnz}\left(\ell_{:, \lambda}\right) \cdot\left\{\operatorname{nnz}\left(\ell_{\mu, 1: \lambda}\right)+\operatorname{nnz}\left(\ell_{\lambda, 1: \lambda}\right)\right\} \lesssim 4^{J-|\lambda|}|\lambda|
$$

Finally, the over-all work of computing the incomplete Cholesky factorization is estimated by summing over all column vectors which yields

$$
\operatorname{cost}(\mathrm{ICF}) \lesssim \sum_{j=0}^{J} 4^{j} 4^{J-j} j=4^{J} J^{2}
$$

i.e., the desired log-linear complexity.

Checking the distance criterion (5.12) for each matrix coefficient, in order to determine the pattern of the incomplete Cholesky factorization, would require $\mathcal{O}\left(N_{J}^{2}\right)$ 
function calls. To realize log-linear complexity, we exploit the underlying tree structure with respect to the supports of the wavelets, to predict the nonzero matrix coefficients. We will call the wavelet $\psi_{\operatorname{son}(\lambda)}$ a son of $\psi_{\lambda}$ if $\Omega_{\operatorname{son}(\lambda)} \subseteq \Omega_{\lambda}$. The following observation, already mentioned in [4], is an immediate consequence of the relation $\mathcal{C}_{j, j^{\prime}} \geq \mathcal{C}_{j+1, j^{\prime}} \geq \mathcal{C}_{j+1, j+1^{\prime}}$

Lemma 5.2. We consider $\Omega_{\mathrm{son}(\lambda)} \subseteq \Omega_{\lambda}$ and $\Omega_{\mathrm{son}(\mu)} \subseteq \Omega_{\mu}$. If

$$
\operatorname{dist}\left(\Omega_{\lambda}, \Omega_{\mu}\right)>\mathcal{C}_{|\lambda|,|\mu|}
$$

then there holds

$$
\begin{aligned}
\operatorname{dist}\left(\Omega_{\operatorname{son}(\lambda)}, \Omega_{\mu}\right) & >\mathcal{C}_{|\lambda|+1,|\mu|}, \\
\operatorname{dist}\left(\Omega_{\operatorname{son}(\lambda)}, \Omega_{\operatorname{son}(\mu)}\right) & >\mathcal{C}_{|\lambda|+1,|\mu|+1} .
\end{aligned}
$$

With the aid of this lemma we have to check the distance criterion only for those coefficients which stem from subdivisions of required coefficients on a coarser level. Therefore, the resulting procedure of checking the distance criterion is still of loglinear complexity:

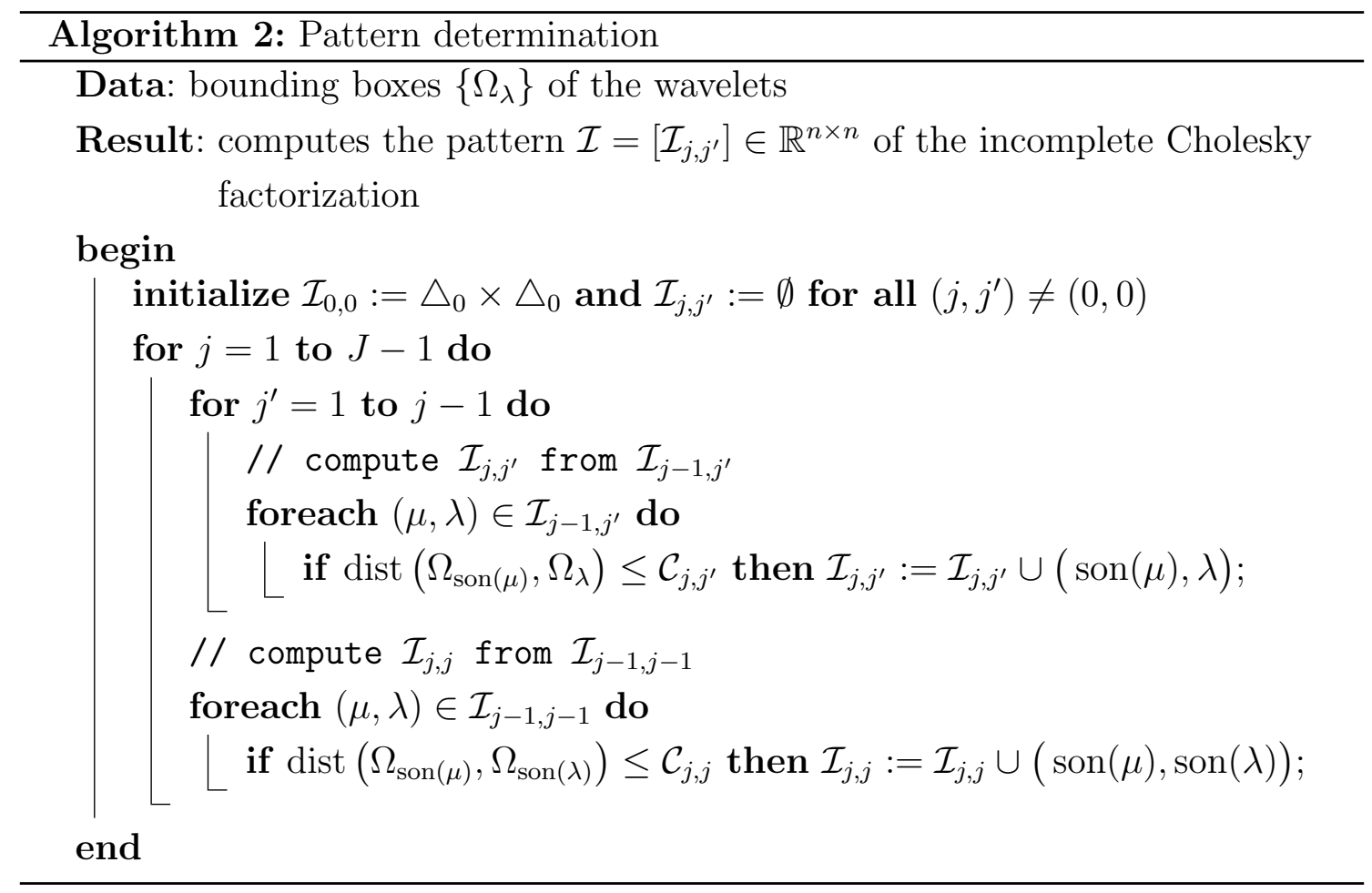




\section{$6 \quad$ Numerical Results}

\subsection{Integral Equations Arising from the Laplace Equation}

To study the quantitative behavior of the incomplete Cholesky factorization, we will consider Symm's integral equation

$$
(\mathcal{V} u)(\mathbf{x})=\int_{\Gamma} \frac{u(\mathbf{y})}{4 \pi\|\mathbf{x}-\mathbf{y}\|} \mathrm{d} \sigma_{\mathbf{y}}=f(\mathbf{x}), \quad \mathbf{x} \in \Gamma
$$

and the hypersingular integral equation

$$
(\mathcal{W} u)(\mathbf{x})=-\frac{\partial}{\partial \mathbf{n}(\mathbf{x})} \int_{\Gamma} \frac{\partial}{\partial \mathbf{n}(\mathbf{y})} \frac{1}{4 \pi\|\mathbf{x}-\mathbf{y}\|} u(\mathbf{y}) \mathrm{d} \sigma_{\mathbf{y}}=g(\mathbf{x}), \quad \mathbf{x} \in \Gamma .
$$

The occurring integral operators satisfy

$$
\mathcal{V}: H^{-1 / 2}(\Gamma) \rightarrow H^{1 / 2}(\Gamma), \quad \mathcal{W}: H^{1 / 2}(\Gamma) / \mathbb{R} \rightarrow H^{-1 / 2}(\Gamma) / \mathbb{R}
$$

Piecewise constant and bilinear wavelets with respectively three and four vanishing moments are used to discretize Symm's integral equation (6.13). The bilinear wavelets are chosen to have double nodes at the edges of the patches. Whereas, the hypersingular integral equation (6.14) is discretized by globally continuous bilinear wavelets with two vanishing moments. Note that the system matrix of the hypersingular operator is positive definite if the underlying bilinear form is modified in accordance with

$$
a(u, v):=(\mathcal{W} u, v)_{L^{2}(\Gamma)}+(u, 1)_{L^{2}(\Gamma)}(1, v)_{L^{2}(\Gamma)},
$$

see e.g. [33].

The related linear systems of equations are solved by the preconditioned CG method up to the absolute accuracy $\varepsilon=10^{-10}$. We compare the standard diagonal scaling (indicated by "diagonal scaling") with the ICF (indicated by "ICF(b)"), where the bandwidth parameter $b$ is chosen as $0,1,2$. In the case $b=0$, the pattern of the ICF contains only coefficients for which the associated wavelets have overlapping supports.

Firstly, we consider $\Gamma$ as the unit sphere, which we represent via six patches. The harmonic function

$$
f(\mathbf{x})=(\mathbf{x}-\mathbf{a})^{T} \mathbf{b} /\|\mathbf{x}\|^{3}, \quad \mathbf{a}=[1.5,0,0]^{T}, \quad \mathbf{b}=[4,2,1]
$$



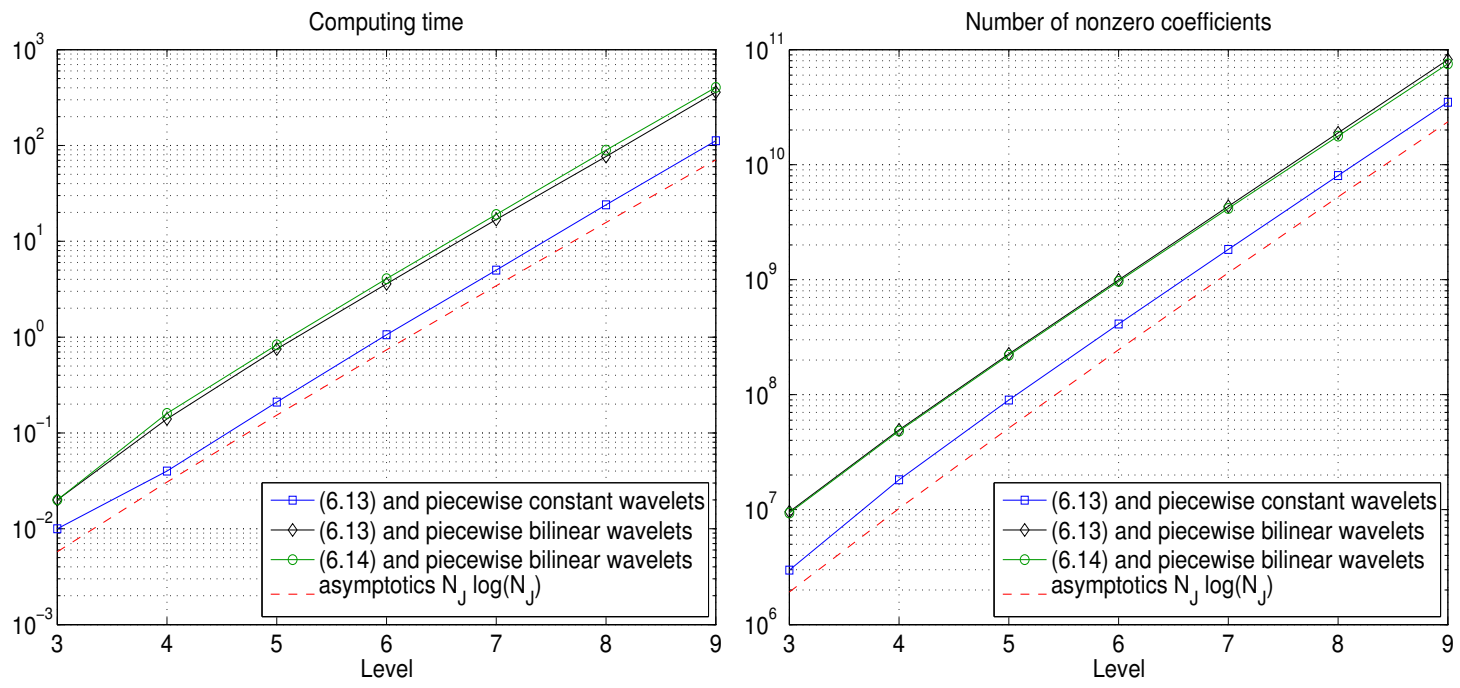

Figure 6.4: Computing time (left) and number of nonzero coefficients (right) of the incomplete Cholesky factorization.

is used as right hand side in (6.13) and $g(\mathbf{x})=(\partial f / \partial \mathbf{n})(\mathbf{x})$ is used as right hand side in (6.14). In Table 6.1, we tabulated the number of iterations, accompanied by the number of nonzero coefficients of the ICF (measured in percent). Secondly, let the boundary $\Gamma$ be the gearwheel shown in Figure 2.1. It is represented via 331 four-sided patches. The right hand side is chosen as in (6.15) but with $\mathbf{a}=\mathbf{0}$. The computational results are tabulated in Table 6.2.

As one figures out of the Tables 6.1 and 6.2, the results are qualitatively the same for both geometries. We observe a drastic decrease of the number of CG-iterations even for the bandwidth parameter $b=0$. The gain of the ICF-preconditioner is at least a factor 10 in the number of iterations compared to the standard diagonal preconditioner. Moreover, an increase of the bandwidth parameter $b$ decreases the number of iterations, which, however, has to be paid by an increase of the number of nonzero coefficients in the ICF.

The computing times of the ICF with respect to the sphere and $b=0$ are found in the left plot of Figure 6.4. For all three cases under consideration, we observe the asymptotic rate $N_{J} \log \left(N_{J}\right)$ (indicated by the dashed line). This is better than the rate $N_{J} \log ^{2}\left(N_{J}\right)$ which has been proven in Theorem 5.1. The number of nonzero coefficient scales also like $N_{J} \log \left(N_{J}\right)$ which however is expected. 


\begin{tabular}{|c|c|c|c|c|c|}
\hline \multicolumn{6}{|c|}{ (6.13) solved by piecewise constant wavelets $(d, \widetilde{d})=(1,3)$} \\
\hline$J$ & $N_{J}$ & $\begin{array}{l}\text { diagonal } \\
\text { scaling }\end{array}$ & $\operatorname{ICF}(0.0)$ & $\mathrm{ICF}(1.0)$ & $\operatorname{ICF}(2.0)$ \\
\hline 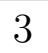 & 384 & 45 & (20) & (32) & (46) \\
\hline 4 & 1536 & 60 & $(7.7)$ & (13) & $(17)$ \\
\hline 5 & 6144 & 74 & $(2.4)$ & (3.9) & (5.7) \\
\hline 6 & 24576 & 86 & $12 \quad(0.68)$ & $(1.2)$ & (1.7) \\
\hline 7 & 98304 & 91 & $12 \quad(0.19)$ & $11 \quad(0.33)$ & $6 \quad(0.50)$ \\
\hline 8 & 393216 & 108 & $13(0.052)$ & $11 \quad(0.093)$ & $7 \quad(0.14)$ \\
\hline 9 & 1.6 Mio. & 117 & $13(0.014)$ & $11 \quad(0.026)$ & $7 \quad(0.039)$ \\
\hline \multicolumn{6}{|c|}{ (6.13) solved by piecewise bilinear wavelets $(d, \widetilde{d})=(2,4)$} \\
\hline 3 & 486 & 93 & $(41)$ & $(62)$ & $(72)$ \\
\hline 4 & 1734 & 104 & $(16)$ & $(27)$ & $(35)$ \\
\hline 5 & 6534 & 1 & $(5.3)$ & $(9.1)$ & $(14)$ \\
\hline 6 & 25350 & 111 & $(1.5)$ & $(2.8)$ & $(4.0)$ \\
\hline 7 & 9984 & & $13 \quad(0.44)$ & $(0.80)$ & $(1.2)$ \\
\hline 8 & 396294 & 125 & $14 \quad(0.12)$ & $(0.22)$ & $6 \quad(0.34)$ \\
\hline 9 & 1.6 Mio. & 132 & $14 \quad(0.033)$ & $9 \quad(0.062)$ & $7 \quad(0.094)$ \\
\hline \multicolumn{6}{|c|}{ (6.14) solved by piecewise bilinear wavelets $(d, \widetilde{d})=(2,2)$} \\
\hline 3 & 386 & 47 & $(63)$ & $(70)$ & $(78)$ \\
\hline 4 & 1538 & 56 & $(20)$ & $(26)$ & $(33)$ \\
\hline 5 & 6146 & 59 & $(5.8)$ & $(8.2)$ & $(11)$ \\
\hline 6 & 24578 & 63 & $(1.6)$ & $(2.4)$ & $(3.3)$ \\
\hline 7 & 98306 & 03 & $(0.43)$ & $(0.68)$ & $4 \quad(0.95)$ \\
\hline 8 & 393218 & 63 & $(0.11)$ & $(0.19)$ & $4 \quad(0.27)$ \\
\hline 9 & 1.6 Mio. & 64 & $\begin{array}{ll}6 & (0.030)\end{array}$ & $5 \quad(0.051)$ & $4 \quad(0.075)$ \\
\hline
\end{tabular}

Table 6.1: Numerical results with respect to the sphere. 


\begin{tabular}{|c|c|c|cc|cc|cc|}
\hline \multicolumn{7}{|c|}{$(6.13)$ solved by piecewise constant wavelets $(d, \widetilde{d})=(1,3)$} \\
\hline$J$ & $N_{J}$ & $\begin{array}{c}\text { diagonal } \\
\text { scaling }\end{array}$ & $\operatorname{ICF}(0.0)$ & $\operatorname{ICF}(1.0)$ & $\operatorname{ICF}(2.0)$ \\
\hline 3 & 18560 & 163 & 16 & $(1.8)$ & 13 & $(2.9)$ & 11 & $(4.3)$ \\
4 & 74240 & 185 & 17 & $(0.57)$ & 13 & $(0.90)$ & 11 & $(1.3)$ \\
5 & 296960 & 198 & 18 & $(0.16)$ & 14 & $(0.25)$ & 12 & $(0.36)$ \\
6 & 1.2 Mio. & 213 & 20 & $(0.043)$ & 14 & $(0.068)$ & 12 & $(0.10)$ \\
\hline \multicolumn{7}{|c|}{$(6.13)$ solved by piecewise bilinear wavelets $(d, \widetilde{d})=(2,4)$} \\
\hline 3 & 27216 & 205 & 11 & $(2.4)$ & 8 & $(4.7)$ & 8 & $(8.1)$ \\
4 & 97104 & 199 & 11 & $(0.51)$ & 9 & $(1.3)$ & 8 & $(2.5)$ \\
5 & 365904 & 212 & 12 & $(0.13)$ & 10 & $(0.36)$ & 8 & $(0.72)$ \\
6 & 1.4 Mio. & 236 & 12 & $(0.035)$ & 11 & $(0.10)$ & 9 & $(0.20)$ \\
\hline \multicolumn{7}{|c|}{$6.14)$ solved by piecewise bilinear wavelets $(d, \widetilde{d})=(2,2)$} \\
\hline 3 & 21504 & 209 & 16 & $(8.1)$ & 15 & $(11)$ & 14 & $(13)$ \\
4 & 86016 & 218 & 16 & $(1.2)$ & 15 & $(2.0)$ & 13 & $(3.0)$ \\
5 & 344064 & 228 & 17 & $(0.25)$ & 16 & $(0.49)$ & 13 & $(0.79)$ \\
6 & 1.4 Mio. & 235 & 18 & $(0.065)$ & 16 & $(0.13)$ & 13 & $(0.22)$ \\
\hline
\end{tabular}

Table 6.2: Numerical results with respect to the gearwheel. 


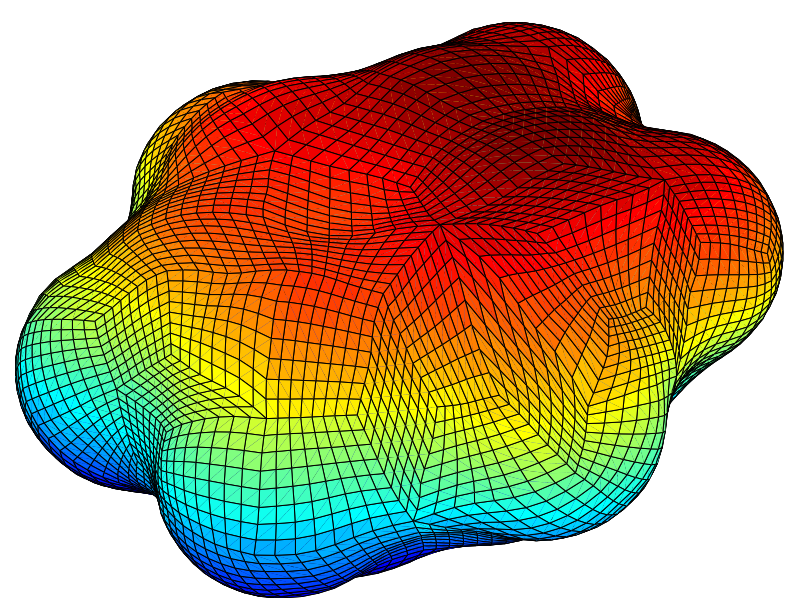

Figure 6.5: Parameterization of the molecular surface of benzene.

\subsection{Polarization Continuum Model}

Continuum solvation models are widely used to model quantum effects of molecules in liquid solutions. In the polarizable continuum model, introduced in [25], the molecule under study (the solute) is located inside a cavity $\Omega$, surrounded by a homogeneous dielectric (the solvent) with dielectric constant $\epsilon \geq 1$. The solutesolvent interactions between the charge distributions which compose the solute and the dielectric are reduced to those of electrostatic origin.

For a given charge $\rho \in H^{-1}(\Omega)$ located inside the cavity, the solute-solvent interaction is expressed by the apparent surface charge $\sigma \in H^{-1 / 2}(\Gamma)$. It is given by the integral equation

$$
\mathcal{V} \sigma=\left(\frac{1+\epsilon}{2}+(1-\epsilon) \mathcal{K}\right)^{-1} \mathcal{N}_{\rho}-\mathcal{N}_{\rho} \quad \text { on } \Gamma:=\partial \Omega
$$

where $\mathcal{V}$ is the single layer potential operator from $(6.13), \mathcal{K}$ is the double layer potential operator

$$
(\mathcal{K} u)(\mathbf{x})=\int_{\Gamma} u(\mathbf{y}) \frac{\langle\mathbf{n}(\mathbf{y}), \mathbf{x}-\mathbf{y}\rangle}{4 \pi\|\mathbf{x}-\mathbf{y}\|} \mathrm{d} \sigma_{\mathbf{y}},
$$

and $\mathcal{N}_{\rho}$ denotes the Newton potential of the given charge

$$
\mathcal{N}_{\rho}(\mathbf{x}):=\int_{\Omega} \frac{\rho(\mathbf{y})}{4 \pi\|\mathbf{x}-\mathbf{y}\|} \mathrm{d} \mathbf{y} .
$$

In the quantum chemical simulations, for example when solving the Hartree-Fock equations in a self consistent field approximation, one has to compute the interaction energies between the different particles. This amounts to the determination of 
different apparent surface charges. Therefore, the fast solution of (6.16) for different right hand sides is indispensable for fast simulations in chemistry.

\begin{tabular}{|c|c|c|cc|cc|cc|}
\hline \multicolumn{7}{|c|}{$(6.16)$ solved by piecewise constant wavelets $(d, \widetilde{d})=(1,3)$} \\
\hline$J$ & $N_{J}$ & $\begin{array}{c}\text { diagonal } \\
\text { scaling }\end{array}$ & $\operatorname{ICF}(0.0)$ & $\operatorname{ICF}(1.0)$ & $\mathrm{ICF}(2.0)$ \\
\hline 3 & 5824 & 48 & 12 & $(2.0)$ & 9 & $(3.9)$ & 8 & $(6.3)$ \\
4 & 23296 & 61 & 12 & $(0.74)$ & 9 & $(1.4)$ & 8 & $(2.1)$ \\
5 & 93184 & 70 & 13 & $(0.23)$ & 9 & $(0.42)$ & 8 & $(0.66)$ \\
6 & 372736 & 81 & 14 & $(0.065)$ & 10 & $(0.12)$ & 7 & $(0.19)$ \\
\hline$(6.16)$ solved by piecewise bilinear wavelets $(d, \widetilde{d})=(2,4)$ \\
\hline 3 & 7371 & 141 & 8 & $(5.2)$ & 6 & $(7.7)$ & 6 & $(11)$ \\
4 & 26299 & 140 & 10 & $(1.8)$ & 7 & $(2.5)$ & 6 & $(3.6)$ \\
5 & 99099 & 133 & 11 & $(0.49)$ & 7 & $(0.78)$ & 6 & $(1.1)$ \\
6 & 384475 & 134 & 14 & $(0.14)$ & 7 & $(0.23)$ & 6 & $(0.33)$ \\
\hline
\end{tabular}

Table 6.3: Numerical results for the polarization continuum model.

We consider benzene as solute and water as solvent $(\epsilon=78.39)$. The associated cavity is represented by 91 four-sided patches, as seen in Figure 6.5. The boundary integral equation (6.16) is discretized by piecewise constant and linear wavelets with 3 and 4 vanishing moments. The solution of the second kind integral equation on the right hand side is well posed and requires thus no preconditioning since $\mathcal{K}$ is compact. In contrast to this, the single layer potential operator needs to be preconditioned.

The given charge $\rho$ consists of point charges which are placed in the nuclei positions. The solution accuracy of the conjugate gradient method is set to $10^{-6}$. In Table 6.3, we tabulated the numerical results. As observed in the previous subsection, the ICF reduces the number of iterations about a factor 10 compared to the standard diagonal preconditioner.

\subsection{Laplace Equation with Stochastic Dirichlet Datum}

The expectation $\mathbb{E}_{u} \in H^{1}(\Omega)$ and the two-point correlation $\operatorname{Cor}_{u} \in H^{1}(\Omega) \otimes H^{1}(\Omega)$ of the solution $u(\omega) \in H^{1}(\Omega)$ to the Laplace equation with stochastic Dirichlet datum

$$
\Delta u(\omega)=0 \text { in } \Omega, \quad u(\omega)=f(\omega) \text { on } \Gamma
$$


is given by the equations

$$
\Delta \mathbb{E}_{u}=0 \text { in } \Omega, \quad \mathbb{E}_{u}=\mathbb{E}_{f} \text { on } \Gamma
$$

and

$$
\begin{aligned}
(\Delta \otimes \Delta) \operatorname{Cor}_{u} & =0 & & \text { in } \Omega \times \Omega, \\
(\Delta \otimes i d) \operatorname{Cor}_{u} & =0 & & \text { in } \Omega \times \Gamma, \\
(i d \otimes \Delta) \operatorname{Cor}_{u} & =0 & & \text { in } \Gamma \times \Omega, \\
\operatorname{Cor}_{u} & =\mathrm{Cor}_{f} & & \text { on } \Gamma \times \Gamma,
\end{aligned}
$$

see $[28,33]$. Note that the two-point correlation of $u$ is a high-dimensional object which lives in $\mathbb{R}^{6}$.

Having a low-rank approximation of the Dirichlet datum's two point-correlation at hand,

$$
\mathrm{Cor}_{f} \approx \sum_{k=1}^{m} \theta_{k} \otimes \theta_{k} \in H^{1 / 2}(\Gamma) \otimes H^{1 / 2}(\Gamma),
$$

then the solution's two point correlation is given by

$$
\mathrm{Cor}_{u} \approx \sum_{k=1}^{m} \eta_{k} \otimes \eta_{k}
$$

with $\eta_{k} \in H^{1}(\Omega)$ solving the Laplace equation

$$
\Delta \eta_{k}=0 \text { in } \Omega, \quad \eta_{k}=\theta_{k} \text { on } \Gamma .
$$

Here, the Neumann datum $\partial \eta_{k} / \partial \mathbf{n} \in H^{-1 / 2}(\Gamma)$ is computed from the Dirichlet datum $\theta_{k} \in H^{1 / 2}(\Gamma)$ by the Dirichlet-to-Neumann map

$$
\mathcal{V} \frac{\partial \eta_{k}}{\partial \mathbf{n}}=\left(\frac{1}{2}-\mathcal{K}\right) \theta_{k}
$$

where $\mathcal{V}$ and $\mathcal{K}$ denotes the single and double layer potential operator (6.13) and (6.17), respectively. Thus, having solved (6.21) for all $k=1,2 \ldots, m$, the two-point correlation $\mathrm{Cor}_{u}$ of the solution $u$ in a point $(\mathbf{x}, \mathbf{y}) \in \Omega \times \Omega$ is given by the representation formula

$$
\operatorname{Cor}_{u}(\mathbf{x}, \mathbf{y})=\sum_{k=1}^{m} \nu_{k}(\mathbf{x}) \cdot \nu_{k}(\mathbf{y})
$$

with

$$
\nu_{k}(\mathbf{x}):=\int_{\Gamma} \frac{\partial \eta_{k}}{\partial \mathbf{n}}(\mathbf{z}) \frac{1}{4 \pi\|\mathbf{x}-\mathbf{z}\|} \mathrm{d} \sigma_{\mathbf{z}}-\int_{\Gamma} \theta_{k}(\mathbf{z}) \frac{\langle\mathbf{n}(\mathbf{z}), \mathbf{x}-\mathbf{z}\rangle}{4 \pi\|\mathbf{x}-\mathbf{z}\|} \mathrm{d} \sigma_{\mathbf{z}} .
$$




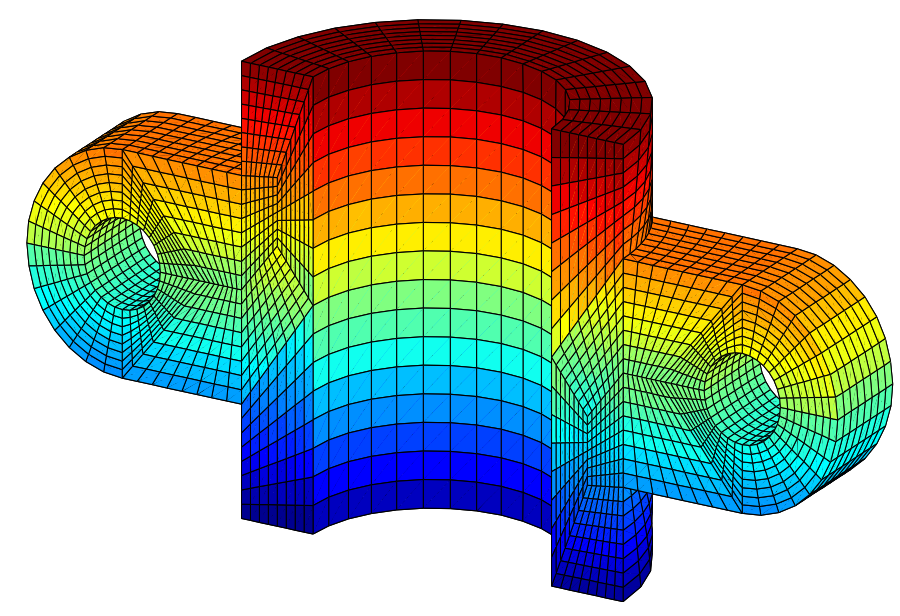

Figure 6.6: The parameterization of a pipe clamp.

We will consider two-point correlation kernels from the Matérn family [10, 24], namely

$$
\begin{aligned}
& k_{3 / 2}(r)=\left(1+\frac{\sqrt{3} r}{\ell}\right) \exp \left(-\frac{\sqrt{3} r}{\ell}\right) \\
& k_{5 / 2}(r)=\left(1+\frac{\sqrt{5} r}{\ell}+\frac{5 r^{2}}{3 \ell^{2}}\right) \exp \left(-\frac{\sqrt{5} r}{\ell}\right)
\end{aligned}
$$

where $r=\|\mathbf{x}-\mathbf{y}\|$ denotes the spatial distance of the points $\mathbf{x}, \mathbf{y} \in \Omega$ and $\ell>0$ is the correlation length. For the computations, we consider $\Omega$ as the pipe clamp seen in Figure 6.6, whose surface $\Gamma$ is represented by 66 four-sided patches. The Dirichlet-toNeumann map (6.21) is discretized by 67584 piecewise constant and 71874 piecewise bilinear boundary elements, respectively. The low-rank approximation (6.19) is determined by the use of a pivoted Cholesky factorization as proposed in [12]. The absolute error of the low-rank approximation is set to $\varepsilon=0.001$. The resulting rank $m$ is found in Table 6.4. It depends on the correlation kernel's smoothness [31] and correlation length $\ell$.

The computation of the ICF for $b=1.0$ consumes 320 seconds in case of the piecewise constant wavelets and 633 seconds in case of the piecewise bilinear wavelets. We mostly need only 3 iterations per CG-solve with the ICF-preconditioning instead of about 70 iterations (in case of piecewise constants) and 200 iterations (in case of piecewise bilinears), respectively, per CG-solve with the diagonal preconditioning. Thus, we save per CG-solve about $50 \%$ of the computing time in case of piecewise constants and about $80 \%$ of the computing time in case of piecewise bilinears. As can be seen from Table 6.4, we thus save up to $80 \%$ of the over-all computing time 


\begin{tabular}{|c|c|c|c|c|c|c|c|}
\hline \multicolumn{8}{|c|}{$(6.21)$ solved by piecewise constant wavelets $(d, \widetilde{d})=(1,3)$} \\
\hline \multicolumn{4}{|c|}{ Matérn kernel $k_{3 / 2}(r)$} & \multicolumn{4}{|c|}{ Matérn kernel $k_{5 / 2}(r)$} \\
\hline$\ell$ & rank & $\begin{array}{l}\text { diagonal } \\
\text { scaling }\end{array}$ & $\mathrm{ICF}(1.0)$ & $\ell$ & rank & $\begin{array}{c}\text { diagonal } \\
\text { scaling }\end{array}$ & $\operatorname{ICF}(1.0)$ \\
\hline 1 & 167 & $781 \mathrm{sec}$ & $733 \mathrm{sec}$ & 0.5 & 162 & $1006 \mathrm{sec}$ & $760 \mathrm{sec}$ \\
\hline 0.75 & 296 & $1376 \mathrm{sec}$ & $1047 \mathrm{sec}$ & 0.375 & 286 & $1400 \mathrm{sec}$ & $1039 \mathrm{sec}$ \\
\hline 0.5 & 668 & $3072 \mathrm{sec}$ & $1945 \mathrm{sec}$ & 0.25 & 624 & $3038 \mathrm{sec}$ & $1879 \mathrm{sec}$ \\
\hline 0.25 & 2631 & 14222 secs & $6888 \mathrm{sec}$ & 0.125 & 2441 & $14308 \mathrm{sec}$ & $6478 \mathrm{sec}$ \\
\hline \multicolumn{8}{|c|}{$(6.21)$ solved by piecewise bilinear wavelets $(d, \widetilde{d})=(2,4)$} \\
\hline \multicolumn{4}{|c|}{ Matérn kernel $k_{3 / 2}(r)$} & \multicolumn{4}{|c|}{ Matérn kernel $k_{5 / 2}(r)$} \\
\hline$\ell$ & rank & $\begin{array}{l}\text { diagonal } \\
\text { scaling }\end{array}$ & $\operatorname{ICF}(1.0)$ & $\ell$ & rank & $\begin{array}{c}\text { diagonal } \\
\text { scaling }\end{array}$ & $\operatorname{ICF}(1.0)$ \\
\hline 1 & 170 & $3123 \mathrm{sec}$ & $1758 \mathrm{sec}$ & 0.5 & 165 & $2722 \mathrm{sec}$ & $1728 \mathrm{sec}$ \\
\hline 0.75 & 309 & $5544 \mathrm{sec}$ & $2230 \mathrm{sec}$ & 0.375 & 283 & $4704 \mathrm{sec}$ & $2114 \mathrm{sec}$ \\
\hline 0.5 & 665 & $11758 \mathrm{sec}$ & 3408 sec & 0.25 & 627 & $11391 \mathrm{sec}$ & 3217 sec \\
\hline 0.25 & 2651 & $39547 \mathrm{sec}$ & $8392 \mathrm{sec}$ & 0.125 & 2457 & $43340 \mathrm{sec}$ & $8264 \mathrm{sec}$ \\
\hline
\end{tabular}

Table 6.4: Numerical results of the stochastic boundary value problem.

for the $m$ solves of (6.21) although the ICF has to be determined first.

\section{Conclusion}

In the present paper, we proposed a new wavelet preconditioning by an ICF with respect to a pattern which takes into account all wavelet-wavelet interactions. The computational complexity of the preconditioner is log-linear. In all numerical tests, the ICF reduces the number of iterations of the preconditioned CG method by at least a factor of 10 in comparison with the standard wavelet preconditioner.

\section{References}

[1] G. Beylkin, R. Coifman, and V. Rokhlin. The fast wavelet transform and numerical algorithms. Comm. Pure and Appl. Math., 44:141-183, 1991. 
[2] C. Carstensen, M. Kuhn, and U. Langer. Fast parallel solvers for symmetric boundary element domain factorization methods. Numer. Math., 79:321-347, 1998.

[3] W. Dahmen. Wavelet and multiscale methods for operator equations. Acta Numerica, 6:55-228, 1997.

[4] W. Dahmen, H. Harbrecht, and R. Schneider. Compression techniques for boundary integral equations. Optimal complexity estimates. SIAM J. Numer. Anal., 43:2251-2271, 2006.

[5] W. Dahmen and A. Kunoth. Multilevel preconditioning. Numer. Math., 63:315344, 1992.

[6] W. Dahmen, S. Prößdorf, and R. Schneider. Multiscale methods for pseudodifferential equations on smooth closed manifolds. In C.K. Chui, L. Montefusco, and L. Puccio, editors, Proceedings of the International Conference on Wavelets: Theory, Algorithms, and Applications, pages 385-424, 1994.

[7] L. Greengard and V. Rokhlin. A fast algorithm for particle simulation. J. Comput. Phys., 73:325-348, 1987.

[8] W. Hackbusch. A sparse matrix arithmetic based on $\mathcal{H}$-matrices. Part I: Introduction to $\mathcal{H}$-matrices. Computing, 64:89-108, 1999.

[9] W. Hackbusch and Z.P. Nowak. On the fast matrix multiplication in the boundary element method by panel clustering. Numer. Math., 54:463-491, 1989.

[10] M.S. Handcock and J.R. Wallis. An approach to statistical spatial-temporal modeling of meteorological fields. J. Amer. Statist. Assoc., 89(426):368-390, 1994.

[11] H. Harbrecht. A Newton method for Bernoulli's free boundary problem in three dimensions. Computing, 82:11-30, 2008.

[12] H. Harbrecht, M. Peters, and R. Schneider. On the low-rank approximation by the pivoted Cholesky decomposition. Appl. Numer. Math., 62(4):428-440, 2012.

[13] H. Harbrecht and T. Hohage. A Newton method for reconstructing non starshaped domains in electrical impedance tomography. Inverse Probl. Imaging, 3(2):353-371, 2009. 
[14] H. Harbrecht and M. Randrianarivony. Wavelet BEM on molecular surfaces. Parametrization and implementation. Computing, 86(1):1-22, 2009.

[15] H. Harbrecht and M. Randrianarivony. From Computer Aided Design to wavelet BEM. Comput. Vis. Sci., 13(2):69-82, 2010.

[16] H. Harbrecht and M. Randrianarivony. Wavelet BEM on molecular surfaces. Solvent excluded surfaces. Computing, 92(4):335-364, 2011.

[17] H. Harbrecht, F. Paiva, C. Pérez, and R. Schneider. Wavelet preconditioning for the coupling of FEM-BEM. Num. Lin. Alg. Appl., 10:197-222, 2003.

[18] H. Harbrecht and R. Schneider. Biorthogonal wavelet bases for the boundary element method. Math. Nachr., 269-270:167-188, 2004.

[19] H. Harbrecht and R. Schneider. Wavelet Galerkin schemes for boundary integral equations. Implementation and quadrature. SIAM J. Sci. Comput., 27(4):1347$1370,2006$.

[20] H. Harbrecht and R. Stevenson. Wavelets with patchwise cancellation properties. Math. Comput., 75(256):1871-1889, 2006.

[21] N. Heuer, M. Maischak, and E. Stephan. Preconditioned minimum residual iteration for the h-p-version of the coupled FEM/BEM with quasi-uniform meshes. Linear Algebra Appl., 6:435-456, 1999.

[22] J. Hoschek and D. Lasser. Grundlagen der geometrischen Datenverarbeitung. Teubner, Stuttgart, 1989.

[23] S. Jaffard. Wavelet methods for fast resolution of elliptic equations. SIAM J. Numer. Anal., 29:965-986, 1992.

[24] B. Matérn Spatial Variation. Second edition. Lecture Notes in Statistics, vol. 36, Springer, Berlin, 1986.

[25] S. Miertuš, E. Scrocco, and J. Tomasi. Electrostatic interaction of a solute with a continuum. A direct utilization of ab initio molecular potentials for the prevision of solvent effects. Chem. Phys. 55:117-129, 1981.

[26] C. Lage and C. Schwab. A wavelet-Galerkin boundary element method on polyhedral surfaces in $\mathbf{R}^{3}$. Numerical treatment of multi-scale problems (Kiel, 1997), pages 104-118, Notes Numer. Fluid Mech. vol. 70, Vieweg, Braunschweig, 1999. 
[27] T. von Petersdorff and C. Schwab. Wavelet approximation for first kind integral equations on polygons. Numer. Math., 74:479-519, 1996.

[28] T. von Petersdorff and C. Schwab. Sparse wavelet methods for operator equations with stochastic data. Applications of Mathematics 51(2):145-180, 2006.

[29] S. Sauter and C. Schwab. Boundary element methods. Springer, BerlinHeidelberg, 2010.

[30] G. Schmidlin and C. Schwab. Wavelet Galerkin BEM on unstructured meshes by aggregation. Multiscale and multiresolution methods, pages 359-378, Lect. Notes Comput. Sci. Eng., vol. 20, Springer, Berlin, 2002.

[31] C. Schwab and R. Todor. Karhunen-Loéve approximation of random fields by generalized fast multipole methods. J. Comput. Phys., 217:100-122, 2006.

[32] R. Schneider. Multiskalen- und Wavelet-Matrixkompression: Analysisbasierte Methoden zur Lösung großer vollbesetzter Gleichungssysteme. Teubner, Stuttgart, 1998.

[33] O. Steinbach. Numerical approximation methods for elliptic boundary value problems. Finite and boundary elements. Springer, New York, 2008. 